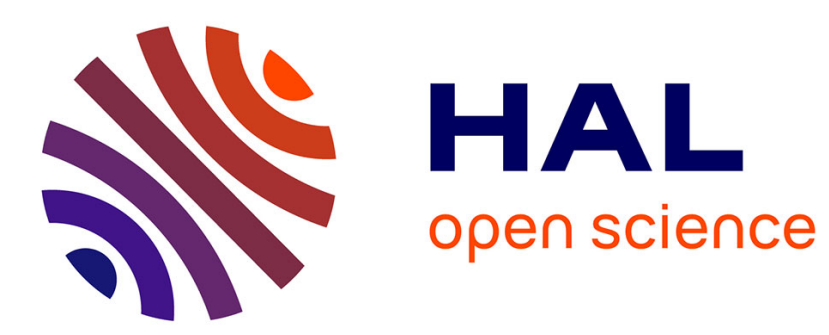

\title{
An investigation of self-organization in ad-hoc networks
}

\author{
Ahmed Akl, Thierry Gayraud, Pascal Berthou
}

\section{To cite this version:}

Ahmed Akl, Thierry Gayraud, Pascal Berthou. An investigation of self-organization in ad-hoc networks. Networking, Sensing and Control (ICNSC), Apr 2011, Delft, Netherlands. pp.1-6. hal00661017

\section{HAL Id: hal-00661017 \\ https://hal.science/hal-00661017}

Submitted on 18 Jan 2012

HAL is a multi-disciplinary open access archive for the deposit and dissemination of scientific research documents, whether they are published or not. The documents may come from teaching and research institutions in France or abroad, or from public or private research centers.
L'archive ouverte pluridisciplinaire $\mathbf{H A L}$, est destinée au dépôt et à la diffusion de documents scientifiques de niveau recherche, publiés ou non, émanant des établissements d'enseignement et de recherche français ou étrangers, des laboratoires publics ou privés. 


\title{
An investigation of self-organization in wireless sensor networks
}

\author{
Ahmed Akl $^{12}$, Thierry Gayraud ${ }^{12}$, and Pascal Berthou ${ }^{12}$ \\ \{akl, gayraud, berthou\}@laas.fr
}

\begin{abstract}
Wireless Sensor Network (WSN) is an emerging special type of ad-hoc wireless networks technology. It is usually designed for special purpose applications. WSN has its own special characteristics that differentiate it from other types of wireless networks. These differences raise new challenges to be overcome; one of them is self-organization.

As in any rising domain, it is essential to specifically define the meaning of new terminologies. The terms self-organizing and self-configuring are an example of such terms that may have overlapping meaning. In this investigation, we tried to make a definition for both terms to specifically determine their role in the WSN domain, and stress on the differences between them.

Consequently, we tried to show the importance of selforganization in enhancing sensor network's performance, and efficient usage of its resources. Thus, we tried to highlight the role of different networking layers in affecting self-organization to be a guideline during WSN design phase. Finally, we highlighted some of the future needs in the WSN domain, and their need for research attention.
\end{abstract}

Key words: Wireless sensor networks; self-organization; selfconfiguration; Ad-hoc networks

\section{INTRODUCTION}

According to Marriam-Webster dictionary[1], the term "Ad hoc" means " formed or used for specific or immediate problems or needs (i.e. ad hoc solutions)". This definition can show the sense of the term "Ad-hoc networks".

Ad-hoc networks are wireless networks where nodes can communicate wirelessly with each other without the need for a fixed infrastructure. This is the most distinguishing feature that differentiates between ad-hoc networks and traditional wireless networks (i.e. LAN, cellular networks). There is no centralized control; nodes are autonomous they can take their own actions depending on network's situation. In other words, they are responsible for determining the way they communicate, organizing themselves, responding to changes that happen to the network due to external or internal factors, etc...

In addition to features inherited from ordinary wireless networks, ad-hoc networks have certain features which are specific to ad-hoc networking. Ad-hoc networks can be composed of heterogeneous nodes; most of these nodes are in continuous mobility. The importance of ad-hoc

\footnotetext{
CNRS; LAAS; 7 avenue du Colonel Roche, F-31077 Toulouse, France

${ }^{2}$ Université de Toulouse; UPS, INSA, ONP, ISAE; LAAS; F-31077 Toulouse, France
}

networking concept is greatly recognised when the nodes are deployed over a large area where a single hop communication is not possible. This situation introduces a challenge to find a technique that provides an appropriate multi-hop routing. Nodes can join or leave the network independently without causing the network to fail.

During the recent years, great developments in electronics and wireless communication allowed researchers to implement miniature sensing devices with wireless communication capabilities. This modern technology satisfies the needs for special type of applications where Wireless Sensor Networks (WSN) can play an important role. This type of networks is useful for applications where rapid deployment is required in areas that lack the appropriate infrastructure to setup the network. It is suitable for environmental measurements, communications in disaster areas, and numerous commercial, personal, and military applications.

WSN is a special type of ad-hoc networks where nodes are smart sensors with scarce resources. They are small in size, have limited computational power, short range communication capabilities, low energy, limited and storage capacity, and usually numerous in number. They differ from the ordinary ad-hoc network's nodes in that they are usually homogenous nodes unless different phenomena are going to be sensed. Many challenges are facing WSN; the main challenges related to WSN implementation are energy conservation, low quality communication, and scalability. Self-organization can help in solving these problems or in the best case to minimize their drawbacks.

Having a quick look on old surveys can give a good image about the great developments that took place in this field. In 1986, T.G.Robertazzi[2] showed the new rising adhoc network technology. At that time they were interested in how to setup the connectivity between nodes, transmission scheduling, self-organization, etc... the concepts of traditional wireless networks were still in mind; using local hubs, backbone networks, gateway were thought to be part of the solution. Nowadays, it is totally different; Ad-hoc networking has its own concepts which proved to be practical and reliable. Although connectivity and transmission scheduling are still important issues, but it became the issue of their performance and reliability. Last but not least, more topics were introduced such as security, and QoS. 


\section{Self-organization Vs Self-COnfiguration}

Self-organization is not a man made concept. Kevin L. Mills[3] showed that it is a natural phenomena that exists in different natural systems. Most of the artificial selforganization techniques were inspired from the natural ones. For example, some anti virus programming concepts were derived from the natural immune system. Natural systems are full of self-organizing mechanisms and concepts that can solve different WSN issues.

The terms self-organization and self-configuration are used interchangeably in the WSN domain to express changes in the current network status to cope with certain environmental change or to enhance network's and/or node's performance[4]. The term self-organization is used more frequently. However, some contributions considered a difference between the two terms[5] to emphasize certain ideas, but there is still a need for a general definition to specifically specify the differences between the two terms. In this section we will try to highlight the differences and propose a clear definition for both of them, so that they can be used unambiguously.

According to Marriam-Webster dictionary[1], "Organization" is derived from the verb "Organize". It has different meanings; those whom we may be interested in are as follows:

- $\quad$ To form into a coherent unity or functioning whole.

- $\quad$ To set up an administrative structure.

- $\quad$ To persuade to associate in an organization.

- To arrange by systematic planning and united effort.

- To arrange elements into a whole of interdependent parts

From the above meanings we can deduce that the verb "Organize" means to arrange different independent entities into a single unity to cooperate together for performing a certain task. Applying the same meaning on the WSN domain, we can define self-organization as "the changes that the node does in its behaviour to cooperate with its neighbours in the network to perform a certain task or achieve a certain goal".

On the other hand, "Configure" was defined as "to set up for operation especially in a particular way"[1]. Applying the same meaning on WSN domain, we can define selfconfiguration as "the changes that the node makes in its parameters to perform certain task".

To sum up, we can say that a node may perform selfconfiguration actions to achieve self-organization that helps the node to have certain behaviour.

For example, if there is an environmental change that causes frames to collide frequently, then each node must be self-organized to overcome this problem in order to minimize power loses. To achieve this behaviour, the node starts to configure its MAC protocol to control the number of sent frames. In this case, we can say that self- configuration had lead to self-organization.

In other situations, self-organization can be achieved without self-configuration. If we considered the case when a node detects a weakness in the received signal due to moving in a certain direction, then it starts to change its direction to keep the signal. This happens without setting up any internal changes, so its behaviour (i.e. self-organization) was changed without any changes in its internal parameters (i.e. self-configuration). This assumption is greatly dependent on the level of abstraction when considering selfconfiguring parameters. In other words, do we consider changes in the values that cause alteration in direction as being changes in configuration or not.

\section{SELF-ORGANIZATION ARCHITECTURAL REQUIREMENTS}

In WSN domain the term self-organization is tightly coupled with routing protocols, so the term self-organizing may be confused with the term routing. Routing is the action of relaying data from one node to another through a communication path. Routing decision may be centralized or decentralised depending on the network architecture. Selforganization is the action of reorienting network nodes. This reorientation can be due to a change in location, hardware or software configuration to change the role that a node plays in the network. The decision of self-organizing is taken by the node itself according to the surrounding environment.

Self-organization is of great importance to manage and save the node's scarce resources. Power consumption can be greatly reduced when transmission range is efficiently managed. In addition, node's location can dramatically affect the choice of an efficient transmission range. Also, it has an influence on network traffic where it can reduce congestion in locations with high nodes density.

An important issue in WSN is that system requirements usually conflict with its physical limitations. A WSN is usually used for gathering and sending data. At the same time, WSN suffers from limited resources such as power source and hardware limitations[6], and limited computational capabilities that needs specific application structure[7]. Further more, WSN are used in different applications ranging from civil[8] and environmental[9] applications to military[10] ones. This wide range of applications needs different system's architectural requirement. It is of a great importance to try to define a general basic architecture and try to find out its requirements. Different application's requirements can be added to this generic architecture to obtain the desired outcome. In this section, we are interested in showing general structural requirements for MAC, network, transport, and application layers that make them suitable for the WSN environment.

\section{A. MAC Layer}

According to the search done by Nait-Abdesselam[11], it was stated that MAC protocols can be categorized into two 
groups, Time Division Multiplexing Access (TDMA), and Carrier Sense Multiple Access (CSMA). The former is basically a technique that allows nodes to share a communication media by synchronising them to share available time slots. Its scheduling nature helps to reduce power losses due collisions. However, there still exists the non ignorable synchronisation overhead. The later is dependent on an agreement between the sender and receiver to share the media instead of waiting for time slots. However, there are losses due to collision, idle listening, and overhearing.

Areas with high density of nodes suffer from congestion due to sharing of same communication medium. Further more, recent improvements in hardware raised the importance of finding appropriate MAC protocols. Ilker Demirkol[12] introduced a survey for different MAC protocols where critical WSN properties for the design of MAC protocols were highlighted. They were also investigated to show their points of weakness and strength. It was mentioned that communication patterns can be categorized into four patterns, broadcast, convergecast, local gossip, and multicast. The differences between these patterns were discussed. Different attributes of a good MAC protocol were proposed. These attributes include energy efficiency, scalability, and adaptability to network changes. In spite of their importance, other attributes can be considered as secondary attributes when compared with the main goals of a MAC protocol. These attributes can be latency, throughput, and bandwidth utilization.

\section{B. Network layer}

The way data are routed between nodes is of great importance. An efficient routing or placement algorithm can save a lot of lost energy. In a WSN environment, nodes are usually deployed randomly causing unintended node distribution. In such situation, it is usually impossible to establish a single hop communication link; this makes it very important to find out a way to transfer packets through multi-hop path(s) efficiently and accurately. This can minimize the energy lost due to packet losses, and sending packets in inappropriate or obsolete paths.

Most of the routing protocols -proposed for the wireless networks' domain- do not consider limited resources constrain that exists in WSN environment. In addition, traditional addressing scheme doesn't work well in such dynamic environment[13]. A well defined routing protocol can solve these issues, but it is impossible to have a single general design, so it is of great importance to identify different categories of protocols. Al-Karaki[13] proposed a good categorization of WSN routing protocols through different points of view according to network structure, and protocol operation. According to the former view, they can be categorized as flat, hierarchical, and location based routing. According to the latter, they can be categorized as negotiation, multi-path, query, QoS, and coherent based routing. In addition, Yazeed Al-Obaisat[14] showed that protocols can also be categorized according to routing discovery; they can be divided into proactive, reactive and hybrid protocols.

Different surveys agreed on common network layer challenges[13][14][15][16]. Node placement, sensors are deployed in either a deterministic or random way. In either case, the network must keep a link of communication between nodes. Energy saving, routing protocols must try to increase the network's life time by adopting an efficient algorithm to minimize energy required for routing. Scalability, node sensors are usually deployed in numerous numbers, the routing protocol must be able to handle extra nodes joining the network and preserve the required performance even if some nodes left the network (i.e. due to power depletion). Mobility, depending on the application, nodes can be either dynamic or static. These issues must be considered during the protocol design.

In addition, the role that the node plays in the network must be considered during the design. In a heterogeneous network environment specialized nodes can exist. A sensing node may be only able to communicate with a router node where there is no need to communicate with neighbouring sensing nodes. Router nodes can communicate with each other to relay data between different groups of sensing nodes. A sink node can be used to collect data sent by sensing nodes. On the other hand, a homogeneous network can exist where each node can play different roles; it can sense as well as route data.

\section{Transport layer}

On the other hand, transport protocols have there own attributes of interest. Chonggang Wang[17] submitted a survey on transport protocols for WSN. Challenges in using transport protocols in sensor environment such as energyefficiency, quality of service, reliability, and congestion control were mentioned. These challenges (which are due to the especial characteristics of WSN) raises the need to design either new transport protocols or to adapt the existing ones because ordinary transport protocols such as UDP or TCP don't behave well in WSN[18][19]. A guideline for transport protocol design was proposed[17]. This guideline discuses common performance metrics (i.e. reliability, QoS, and fairness), and required functions of transport protocols (i.e. congestion control, and loss recovery) with respect to WSN environment. Chonggang Wangl[18] presented different design issues for transport layer in WSN. In[17] and [18] different types of transport protocols dedicated for WSN were discussed.

WSN are not an isolated network; for certain applications it is necessary to be connected to other types of networks such as internet or a database. Most of these networks, if not all, are dependent on the TCP protocol. Unfortunately, TCP doesn't behave well in WSN. So, if this type of connection is really needed, then adapting the transport layer should be 
considered to be able to establish this type of connection. Chonggang Wangl[18] presented the disadvantages of using TCP and UDP, with respect to a WSN environment, which were originally designed for wired, or in the best cases for wireless networks. Mirko R. Kosanovic[19] proposed a solution to overcome this type of problems in order to connect WSN to other TCP networks.

\section{Application layer}

From the first sight, it seems that self-organization is only dependent on the lower communication layers. In fact, a WSN application layer can have a considerable impact on self-organization and node's performance. The basic software level is the Operating System (OS). It is the responsibility of the OS to manage the node's resources such as, memory, CPU, and communication capabilities. Requirements for such OS were introduced by Yao minghai[20].

When the node's structure is too complex, the applications may need a solution to hide this complexity for easier development process. In this case, intermediate software is required to handle the situation; this type of software is called middleware. Middleware was presented in a survey by Miao-Miao Wang[21], where different issues were discussed including a study for the topic to determine the challenges, the services required, and provide a reference model for determining the required functionalities and services. In addition, he showed the current work related to this topic, as well as proposing a way to organize the relations between the middleware features to give a better understanding for the issue.

Although different programming models were introduced for the networking domain, but programming for WSN applications needs extra attention due to the different constrains that exist in WSN environment. Ryo Sugihara[22], introduced a comprehensive survey for representing the programming requirements, showing the challenges that arises due to limited resources, and introducing available programming models of WSN.

Another issue that shows the importance of application layer for self-organization is software update. When an environmental change occurs, such as the existence of new type of nodes, extra phenomena to be measured, and a change in the node's task; a software update may be vital for the node to cope with these changes. S.Brown[23], introduced a survey for software update in WSN. It showed different issues such as the effect of update on performance, security, and energy saving, as well as known research categories for software update.

\section{SELF-ORGANIZATION TECHNIQUES}

In many applications especially those interested in measuring natural phenomena or rescue missions, sensor nodes are randomly spread in a large geographical area. This situation leads to the need for an organizing technique to reorganize the nodes to efficiently utilize them.

Recently, different techniques were investigated to show the importance and effectiveness of self-organization. In this part, we will introduce techniques related to topological and power conservation self-organization.

\section{A. Topological self-organization}

WSN is a special type of networks where not all topologies can be efficiently implemented. Star, mesh, and cluster hierarchy are the most chosen topologies for WSN. Akhilesh Shrestha[24] introduced a comparison between the three topologies. It was shown that mesh topology takes the highest organizing time. Star topology takes the lowest time and is the least complex. In the cluster approach, organization time is dependent on the time taken for clusters initiation. It was stated that for star topology, adding more nodes has a great impact on network's performance. This is due to the increased overhead on the base node, and the increased congestion in areas of high nodes' density. Further more, most nodes have no direct connection to the base node; this increases the network overhead and creates a scalability problem. On the other hand, mesh networks determines routes depending on link quality between neighbours. Theoretically it can be extended to any size, but due to performance limitations it is suitable for medium sized networks; while faces scalability problems with large networks or networks of high density areas. In contrast, nodes in a cluster based network neighbouring are managed by their cluster head node. This allows a rapid and periodic reformation of the network hierarchy in cases of node(s) failure, node addition, and changes due external factors (i.e. environmental changes).

In some applications, it is required to disperse a huge number of sensing nodes in a large area. This may lead to a different nodes' densities distribution within the working area. TheinLai Wong[25] proposed an algorithm for reorganizing the initial random nodes distribution to enhance the coverage of the sensing network while minimizing energy consumption by minimizing the amount of movements depending on local information only. In this technique a concept of virtual forces was applied to reorganize the nodes. If the distance between two nodes is less than certain limit, then there will be a repulsive force between them. If the distance is more than certain limit, then there will be an attraction force between them. The node will not move if the distance is equal to the threshold. This mechanism will introduce unneeded movements that will consume more power, so a random "Back-off" delay is initiated at each node at the beginning to minimize this effect. This process is repeated until the network settles.

Sungyun Park[26] work was based on the clustering concept. The idea is to minimize the number of network's clusters where nodes are participating. Each cluster has a single cluster head, and nodes are only communicating with their cluster head. A clustering algorithm was introduced to 
merge overlapping clusters and remove redundant cluster heads. A caching strategy was suggested to enhance the algorithm's performance. The algorithm was compared with two other clustering algorithms, Algorithm for Cluster Establishment (ACE), and Self Organizing Sensor network algorithm (SOS). The proposed optimization algorithm showed better results and more stability than the other two algorithms.

\section{B. Power management self-organization}

The sensing nodes have a limited energy supply, so lowpower operation is a must. The lifetime of a sensor network is generally limited to the battery lifetime of sensor nodes. Energy consumption occurs in sensing, data processing, and communication. In this part we are concerned with power savings due communication self-organization. Apart of node movements, communication is the most intensive power consuming activity.

Sooksan Panichpapiboon[27] stated that high power transmissions decreases the node's life time as well as introducing unnecessary interference. It is one of the main goals of the network to preserve connectivity. Using low transmission power may cause simultaneous disconnections in the networks, while using excessive power will increase interference due to sharing of same radio channel. The author considered QoS, with respect to Bit Error Rate (BER), to indicate the presence or absence of a communication link. Generally speaking, it is usually assumed that a communication link is present between two nodes if they are within the transmission range of each other; according to the QoS criteria this may not be true, because a link between two nodes may not satisfy the required QoS constrain. The optimal transmission power was evaluated analytically for a regular topology, and using simulation for random topology. The proposed optimal transmitting power is dependent on the used MAC and routing protocols, but the same concept can be applied to other protocols.

The O-MAC protocol was introduced by Farid NaitAbdesselam[11]. It is specifically designed for WSN. It was inspired from the S-MAC protocol. The aim of the proposed protocol is to minimize power losses due to collisions, overhearing, and idle listening. This leads to a prolonged life time of the network. It is based on CSMA technique to minimize collisions. Nodes within the transmission range who are not concerned with the current transmission can enter a sleeping mode to save their power. Their neighbors are informed with their status in order not to send unnecessary data. The protocol was tested with an OPNET simulation and its performance was compared with S-MAC and IEEE802.11 protocols. The comparison was made for three topologies with respect to the metrics, energy consumption, volume of data collected at the $\operatorname{sink}(\mathrm{s})$ node, and the ratio of the data collection to the energy consumption (to indicates the energy efficiency factor). The results showed that O-MAC has a good performance when compared with the other two protocols.

Some WSN localization systems are optimized for indoor applications. Most of these systems provide static anchor nodes and localizing mobile nodes fixed on the object to be observed. This structure helps in estimating the location with high precision. Marcel Baunach[28] introduced a method adapted for localized environments called "HashSlot". This method is suitable for environments where the working area is well defined. It is a TDMA (Time Division Multiplexing Access) technique that depends on the location of anchor nodes within a grid field to dynamically compute transmission slots. These slots are computed by each anchor node without any interaction or communication with other anchor nodes. This method provides a collision free communication environment between one hop multiple resources and single destination. This method was verified using the "Snow Bat" test bed which uses Ultrasonic sensors for distance measurement with an accuracy of $4 \mathrm{~mm}$. It is a reliable system to measure $2 \mathrm{D} / 3 \mathrm{D}$ tracking of mobile objects.

\section{CONCLUSION}

Natural systems had proved to be good competent, more reliable, and fault tolerant. These pre-tested natural systems give confidence in acquiring good results when inspiring techniques derived from them. One of their most interesting features is self-organization.

In WSN domain, self-organization and self-configuration are two different terms which are usually used interchangeably. We thoroughly identified them so that they can be used more precisely in the context.

One of the current features of WSN is that solutions tend to be application dependent; leading to different design concepts and approaches. We believe that, although each network layer can have a sole effect on self-organization, a better performance can be achieved if the global view of all layers were considered, so we showed the role of each network layer to acquire self-organization in order to achieve better understanding as well as being able to evaluate different approaches.

\section{FUTURE NEEDS}

WSN is a new domain that faces a lot of challenges, and can be used in numerous types of applications which are not completely revealed till now. One of these domains is time critical applications where time of data transfer is a great issue, and they need to be zero tolerant for data loss. This type of applications needs to be considered by researchers.

Moreover, some applications need certain level of fault tolerance and reliability. Current WSN designs are mainly concerned with connectivity and power saving without paying attention for reliability issues.

The aim of most WSN design is to achieve certain goal(s) depending on the required application. This way of design 
lacks a standard measure of performance. Determining general metrics for performance can be a new challenge for researchers.

Some traditional concepts such as distributed computing can attract the attention for usage in WSN environment. However, such concepts must be retailored to suit this domain

\section{REFERENCES}

[1] http://www.merriam-webster.com/dictionary.

[2] T.G. Robertazzi and P.E. Sarachik, "Self-organizing Communication Networks," IEEE Communications Magazine, vol. 24, 1986, pp. 28-33

[3] K.L. Mills, "A brief survey of self-organization in wireless sensor networks," Wireless Communication and Mobile Computing, vol. 7, 2007.

[4] J. Ibriq and I. Mahgoub, "Cluster-Based Routing in Wireless Sensor Networks : Issues and Challenges," Proceedings of the International Symposium on Performance Evaluation of Computer and Telecommunication Systems, 2004, pp. 759-766.

[5] J.-liang Lu, F. Valois, D. Barthel, and M. Dohler, "FISCO : a Fully Integrated Scheme of self-Configuration and self-Organization for WSN," WCNC, 2007, pp. 3372-3377.

[6] J. Li, M. Li, and L. Sun, "A Low Power Consumption Implementation for WSN Nodes in Lumber Drying Kiln," IEEE International Conference on Mechatronics and Automation, 2007, pp. 911-916.

[7] M. Kuorilehto, M. MarkoHannikainen, and T.D. Hamalainen, "A Survey of Application Distribution," EURASIP Journal on Wireless Communications and Networking, vol. 5, 2005, pp. 774-788.

[8] J.A. Stankovic, Q. Cao, T. Doan, L. Fang, Z. He, R. Kiran, S. Lin, S. Son, R. Stoleru, and A. Wood, "Wireless Sensor Networks for InHome Healthcare : Potential and Challenges," in High Confidence Medical Device Software and Systems (HCMDSS) Workshop, 2005.

[9] R. Behnke, F. Golatowski, K. Thurow, and D. Timmermann, "Wireless Sensor Networks for Life Science Automation," In International Forum Life Science Automation, 2007.

[10] T. Bokareva, W. Hu, S. Kanhere, B. Ristic, and N.S. Wales, "Wireless Sensor Networks for Battlefield Surveillance," Proceedings of the Land Warefare Conference, 2006.

[11] F. Nait-Abdesselam, B. Bensaou, T. Soete, and K.-L. Hung, "O-MAC : An Organized Energy-Aware MAC Protocol for Wireless Sensor Networks," IEEE International Conference on Communications, 2007, pp. 3648-3653.

[12] I. Demirkol, C. Ersoy, and F. Alagoz, "MAC Protocols for Wireless Sensor Networks : A Survey," IEEE Communications Magazine, 2006, pp. 115-121.

[13] J.N. Al-karaki and A.E. Kamal, "Routing Techniques in Wireless Sensor Networks : A Survey," IEEE wireless Communication, vol. 11, 2004, pp. 6-28.

[14] Y. Al-obaisat and R. Braun, "On Wireless Sensor Networks : Architectures , Protocols , Applications , and Management," Auswireless Conference, 2006.

[15] M.A. Perillo and W.B. Heinzelman, "Wireless Sensor Network Protocols," International Conference On Communication Technology, 2006.

[16] K. Akkaya and M. Younis, "A survey on routing protocols for wireless sensor networks," Elsevier Ad Hoc Networks Journal, vol. 3 , 2005 , pp. 325-349.

[17] C. Wang, K. Sohraby, B. Li, M. Daneshmand, and Y. Hu, "A Survey of Transport Protocols for Wireless Sensor Networks," IEEE Network Magazine, 2006, pp. 34-40.

[18] C. Wangl, K. Sohraby, Y. Hu, B. Li, and W. Tang, "Issues of Transport Control Protocols for Wireless Sensor Networks," International Conference on Communications, Circuits, and systems, 2005, pp. 422-426.

[19] M.R. Kosanovic and M.K. Stojcev, "Implementation of TCP / IP Protocols in Wireless Sensor Networks," Proceedings of the International Scientific Conference on Information, Communication and Energy Systems and Technologies (ICEST), 2007, pp. 143-146.
[20] Y. Ming-Hai and Z. Xiao-Xiao, "Study and Transplant of Operating System for Wireless Sensor Network Node," International Conference on Wireless Communication, Networking, and Mobile Computing, 2007, pp. 2803-2807.

[21] M.-miao Wang, J.-N. Cao, J. Li, and S.K. Das, "Middleware for Wireless Sensor Networks : A Survey," Journal of Computer Science and Technology, vol. 23, 2008, pp. 305-326.

[22] R. Sugihara and R.K. Gupta, "Programming Models for Sensor Networks : A Survey," ACM Transaction On Sensor Networks (TOSN), 2008, pp. 1-27.

[23] S. Brown and C.J. Sreenan, "Updating Software in Wireless Sensor Networks : A Survey," Technical Report UCC-CS-2006-13-07, National University of Ireland, 2006.

[24] A. Shrestha and L. Xing, "A Performance Comparison of Different Topologies for Wireless Sensor Networks," IEEE conference on Technologies for Homeland Security, 2007, pp. 280-285.

[25] T. Wong, T. Tsuchiya, and T. Kikuno, "A Self-organizing Technique for Sensor Placement in Wireless Micro-Sensor Networks," Proceedings of the 18th International Conference on Advanced Information Networking and Applications (AINA), vol. 2, 2004.

[26] S. Park, K. Shin, A. Abraham, and S. Han, "Optimized Self Organized Sensor Networks," Sensors ISSN 1424-8220; CODEN: SENSC9, an international open access Journal, vol. 7, 2007, pp. 730-742.

[27] S. Panichpapiboon, G. Ferrari, and O.K. Tonguz, "Optimal Transmit Power in Wireless Sensor Networks," IEEE Transaction On Mobile Computing, vol. 5, 2006, pp. 1432-1447.

[28] M. Baunach, R. Kolla, and C. M, "A Method for Self-Organizing Communication in WSN Based Localization Systems : HashSlot," 32nd IEEE Conference on Local Computer Networks, 2007, pp. 825832 